\title{
Optimal Sampling Strategies for Therapeutic Drug Monitoring of First-Line Tuberculosis Drugs in Patients with Tuberculosis
}

\author{
Antonia Morita I. Saktiawati ${ }^{1,2,3} \cdot$ Marcel Harkema $^{4} \cdot$ Althaf Setyawan $^{5} \cdot$ Yanri W. Subronto $^{1,3} \cdot$ Sumardi $^{1}$. \\ Ymkje Stienstra $^{6} \cdot$ Rob E. Aarnoutse ${ }^{7}$. Cecile Magis-Escurra ${ }^{8} \cdot J^{\prime}$ os G. W. Kosterink ${ }^{9,10} \cdot$ Tjip S. van der Werf $^{2,6}$. \\ Jan-Willem C. Alffenaar ${ }^{9,11} \cdot$ Marieke G. G. Sturkenboom ${ }^{9}$
}

Published online: 17 April 2019

(c) The Author(s) 2019

\begin{abstract}
Background The 24-h area under the concentration-time curve $\left(\mathrm{AUC}_{24}\right) /$ minimal inhibitory concentration ratio is the best predictive pharmacokinetic/pharmacodynamic (PK/PD) parameter of the efficacy of first-line anti-tuberculosis (TB) drugs. An optimal sampling strategy (OSS) is useful for accurately estimating $\mathrm{AUC}_{24}$; however, OSS has not been developed in the fed state or in the early phase of treatment for first-line anti-TB drugs.

Methods An OSS for the prediction of $\mathrm{AUC}_{24}$ of isoniazid, rifampicin, ethambutol and pyrazinamide was developed for TB patients starting treatment. A prospective, randomized, crossover trial was performed during the first 3 days of treatment in which first-line anti-TB drugs were administered either intravenously or in fasting or fed conditions. The PK data were used to develop OSS with best subset selection multiple linear regression. The OSS was internally validated using a jackknife analysis and externally validated with other patients from different ethnicities and in a steady state of treatment.

Results OSS using time points of 2, 4 and $8 \mathrm{~h}$ post-dose performed best. Bias was $<5 \%$ and imprecision was $<15 \%$ for all drugs except ethambutol in the fed condition. External validation showed that $\mathrm{OSS}_{2-4-8}$ cannot be used for rifampicin in steady state conditions.

Conclusion OSS at 2, 4 and $8 \mathrm{~h}$ post-dose enabled an accurate and precise prediction of $\mathrm{AUC}_{24}$ values of first-line anti-TB drugs in this population.

Trial Registration ClinicalTrials.gov (NCT02121314).
\end{abstract}

\section{Key Points}

Little information is available on sampling strategies in early TB treatment and in different conditions. This study investigated optimal sampling strategies (OSSs) to estimate area under the concentration-time curve from time zero to $24 \mathrm{~h}\left(\mathrm{AUC}_{24}\right)$ of first-line anti-TB drugs in early treatment and in different conditions.

An OSS at 2, 4 and $8 \mathrm{~h}$ post-dose accurately predicted the $\mathrm{AUC}_{24}$ of first-line anti-TB drugs in fasting and fed conditions in early treatment. These OSS equations support the therapeutic drug monitoring programmes aiming to personalize oral dosing for adult TB patients.

Marieke G. G. Sturkenboom

m.g.g.sturkenboom@umcg.nl

Extended author information available on the last page of the article

\section{Introduction}

Tuberculosis (TB) is a major problem worldwide. It is a curable disease but, nevertheless, 1.7 million people died from TB in 2016 [1]. The reported treatment success rate for drug-susceptible TB ranges from 60 to $83 \%$ [1], meaning that healthcare providers encounter treatment failure in daily practice. The World Health Organization (WHO) aims to end the global TB epidemic by 2035 through several pillars and component goals, some of which are intensifying research and innovation by the development of new strategies and optimization of implementation of treatment [1]. Optimization of treatment is imperative to increase the success rate of first-line treatment.

First-line treatment of TB consists of isoniazid, rifampicin, pyrazinamide and ethambutol for the first 2 months, followed by isoniazid and rifampicin for 4 months [2]. Clinical trials and observational studies have shown that low drug exposure of first-line drugs is associated with slow response to treatment, acquired drug resistance, and relapse [3, 4]. 
Several factors, such as malabsorption, pharmacogenetics of N-acetyltransferase 2 (NAT2), intraindividual differences in pharmacokinetics (PK), or food intake, may lead to low drug exposure [4-6]. Treatment optimization can be achieved by increasing adherence [7] or studying new or repurposed drugs $[8,9]$. Another option is better attainment of the pharmacokinetic/pharmacodynamic (PK/PD) parameters that best predict the efficacy of first-line anti-TB drugs, i.e. area under the concentration-time curve over $24 \mathrm{~h}$ (AUC 24) divided by the minimal inhibitory concentration (MIC) [AUC/MIC ratio] [10]. However, actual MICs are often unavailable for first-line anti-TB drugs. Using classification and regression tree (CART) analysis, Pasipanodya et al. showed a clear concentration-effect relationship for the combination therapy of pyrazinamide, rifampicin and isoniazid [3].

Therapeutic drug monitoring (TDM), which measures drug exposure and adjusts the dose to optimize drug exposure, has been proposed to improve treatment response in individual TB patients, especially in patients at high risk of treatment failure or delayed response [11-14]. To calculate drug exposure, i.e. $\mathrm{AUC}_{24}$, a full concentration-time curve should be obtained by sampling up to 10 times during the dosing interval. The burden for both patients and the healthcare system hamper implementation in clinical practice $[15,16]$. Therefore, there is an urgent need for alternative strategies to accurately estimate the $\mathrm{AUC}_{24}$. A limited or optimal sampling strategy (OSS) might be the solution. This method calculates which time points during the dosing interval (usually one to three) are most informative for the accurate prediction of $\mathrm{AUC}_{24}[14,15]$.

Only a few studies in the literature have reported OSS for first-line anti-TB drugs [15-18]; however, these studies mostly addressed administration of a single drug $[15,17,18]$ in the fasting condition [16-18], and no studies examined OSS in the early phase of treatment. Meanwhile, the number of bacilli was high in the early phase of treatment, thus the risk of acquired drug resistance was higher when drug exposure was inadequate [3]. One study described an OSS for multiple anti-TB drugs simultaneously, but, in that study, the time interval was limited to $6 \mathrm{~h}$ and was conducted in the fasting condition [16].

To overcome the limitations of currently available OSS, we aimed to develop an OSS in the early phase of treatment to estimate the $\mathrm{AUC}_{24}$ of all first-line anti-TB drugs in fasting and fed conditions, as well as after intravenous administration.

\section{Methods}

\subsection{Study Design, Population, and Pharmacokinetic Analysis}

To develop the OSS, we used data from an earlier published prospective, randomized, crossover PK study approved by the Institutional Review Board at the Faculty of Medicine, Public Health and Nursing, Universitas Gadjah Mada (UGM), Yogyakarta, Indonesia (KE/FK/626/ EC), and registered at ClinicalTrials.gov (NCT02121314) [19]. From November 2012 to March 2013, 20 newly diagnosed, treatment-naïve adults with drug-susceptible TB received isoniazid $5 \mathrm{mg} / \mathrm{kg}$, rifampicin $10 \mathrm{mg} / \mathrm{kg}$, ethambutol $15 \mathrm{mg} / \mathrm{kg}$ and pyrazinamide $25 \mathrm{mg} / \mathrm{kg}$. One patient was excluded from the study due to vomiting shortly after ingestion of the drugs on both days of oral treatment, and was replaced by inclusion of another subject. The study was performed on the first 3 days of anti-TB treatment. On day 1, all subjects received intravenous treatment of isoniazid $(100 \mathrm{mg} / \mathrm{mL}$ injection; Department of Clinical Pharmacy and Pharmacology, University Medical Center Groningen (UMCG), Groningen, The Netherlands; license number 108964F), rifampicin (rifadin $600 \mathrm{mg}$ injection; Sanofi Aventis, Guildford, UK) and ethambutol (EMBFatol 1000 mg injection; Riemser Arzneimittel AG, Greifswald - Insel Riems, Germany). Isoniazid was administered as a short infusion of $30 \mathrm{~min}$, while rifampicin and ethambutol were infused over $2 \mathrm{~h}$. Pyrazinamide was not available as an injection and was administered as a $500 \mathrm{mg}$ tablet formulation (PT Indofarma, Bekasi, Indonesia) in a single oral dose on day 1.

Participants were randomly assigned to one of the two treatment groups (fasted day 2 and fed day 3, or fed day 2 and fasted day 3). On days 2 and 3, a fixed drug combination containing 75/150/275/400 $\mathrm{mg}$ of isoniazid, rifampicin, ethambutol and pyrazinamide (PT Indofarma) was administered orally. Serial blood samples were collected at 0 (predose), 0.5, 1, 1.5, 2, 2.5, 3, 4, 5, 6 and $8 \mathrm{~h}$ after dosing, and were centrifuged and stored at $-80{ }^{\circ} \mathrm{C}$ in the TB-Microbiology Laboratory of the Faculty of Medicine, Public Health, and Nursing, UGM, Indonesia, until transported on dry ice to The Netherlands, with the provision of a Material Transfer Agreement (UGM-UMCG, and the National Agency of Food and Drug Control, Indonesia; PN.01.06.1.31.11.12.7158). Plasma samples were analysed using validated liquid chromatography-tandem mass spectroscopy (LC-MS/MS) methods in the laboratory of the Department of Clinical Pharmacy and Pharmacology of the UMCG, The Netherlands, as previously described [20,21]. Concentrations at time points 0 on days 2 and 3 also served as the 24-h concentration $\left(\mathrm{C}_{24}\right)$ on days 1 and 2 . The $C_{24}$ on day 3 was calculated using the formula $C_{24}=C_{\max } \times e^{-\beta \times(24-T \max )}$, in which $\beta$ is the first-order elimination rate constant, $\mathrm{C}_{\max }$ is the maximum concentration measured, and $T_{\max }$ is the time to reach $C_{\max }$. Plasma concentrations below the lower limit of quantification were treated as zero. The $\mathrm{AUC}_{24, \text { calculated }}$ was calculated using the log-linear trapezoidal rule in MW\Pharm (Kinfit, version 3.60; Mediware, Groningen, The Netherlands); this 
$\mathrm{AUC}_{24, \text { calculated }}$ represented the best approximation of the true $\mathrm{AUC}_{24}$.

\subsection{Optimal Sampling Strategies}

We conducted multiple linear regression analysis to obtain optimal sampling equations predictive of the $\mathrm{AUC}_{24}$ of each drug in the three different conditions. All possible combinations within the groups of one to three time points were evaluated, i.e. 11 combinations with one time point, 55 combinations with two time points, and 165 combinations with three time points. Assumptions of linear regression were assessed using R, i.e. normality of the dependent variable, linear relationship, error normality, multicollinearity and homoscedasticity.

Using best subset selection, we selected the best-performing OSS for each drug and condition separately, for the simultaneous use of all drugs in each condition, and for the simultaneous use of all drugs after oral administration (either the fasting or fed condition). In addition, we tested the OSS from simultaneous prediction of the fasting and fed conditions following intravenous administration to examine whether the OSS was also suitable for application in intravenous administration.

The average adjusted $R^{2}$ for all combinations comprising one, two, or three samples were calculated to investigate the correlation between the predicted $\mathrm{AUC}_{24}$ (AUC 24,predicted) using multiple linear regression and AUC 24,calculated. With a time resolution of $1 \mathrm{~h}$, the best OSS was selected based on the highest average adjusted $\mathrm{R}^{2}$ combined with an acceptable bias and precision.

\subsection{Validation of the Optimal Sampling Strategy}

To validate the OSS model, residuals for each patient were calculated using a jackknife analysis [16, 22]. All 20 patients were omitted, on a one-by-one basis, and the OSS using best subset selection was calculated for the 19 remaining patients in each scenario, as described above. The $\mathrm{AUC}_{24, \text { predicted }}$ and corresponding residuals were then calculated for the omitted patient.

The following residuals were calculated. To assess potential bias in the predictions, the median prediction error (MPE) and median percentage prediction error (MPPE) were calculated. The MPE was converted into MPPE by dividing it by the $\mathrm{AUC}_{24, \text { calculated }}$ values [22-24]. To assess precision, root median squared prediction error (RMSE) and median absolute percentage prediction error (MAPE) were calculated $[23,25]$. The equations used to calculate these four residuals were as follows [23]:
Bias

1. MPE: median ( $\left.\mathrm{AUC}_{24, \text { predicted }}-\mathrm{AUC}_{24, \text { calculated }}\right)$

2. MPPE: median $\left[100 \% \times\left(\mathrm{AUC}_{24, \text { predicted }}-\mathrm{AUC}\right.\right.$ 24 ,calculated $\left./ \mathrm{AUC}_{24, \text { calculated }}\right]$

Imprecision

1. RMSE: $\sqrt{ }$ median $\left(\mathrm{AUC}_{24, \text { predicted }}-\mathrm{AUC}_{24, \text { calculated }}\right)^{2}$

2. MAPE: median $\left[100 \% \times \mathrm{I}\left(\mathrm{AUC}_{24, \text { predicted }}-\mathrm{AUC}\right.\right.$ 24 ,calculated $) \mathrm{I} / \mathrm{AUC}_{24, \text { calculated }]}$

OSS was considered acceptable if bias and imprecision were smaller than $5 \%$ and $15 \%$, respectively $[15,16,23]$.

A percentage prediction error (PPE), calculated as $\left[100 \% \times\left(\mathrm{AUC}_{24, \text { predicted }}-\mathrm{AUC}_{24, \text { calculated }}\right) / \mathrm{AUC}_{24, \text { calculated }}\right]$, was used to show the performance of OSS equations from each individual $[24,26]$. An acceptable PPE was considered smaller than $15 \%$ [24, 26], and the number of patients with a PPE $<15 \%$ was calculated for the best-performing OSS. Data analysis was carried out using $\mathrm{R}$ version 3.3.2 (The $\mathrm{R}$ Foundation, Austria, Vienna).

\subsection{External Validation}

To test whether the selected OSS was capable of predicting the $\mathrm{AUC}_{24}$ in other patient groups, an external validation group was selected. This external validation group was the population in which Magis-Escurra et al. developed an OSS for the first-line drugs and also moxifloxacin. Study subjects were TB patients admitted to the two Dutch TB referral centres, i.e. Radboud University Medical Centre, Nijmegen, Centre for Chronic Diseases Dekkerswald, Groesbeek, The Netherlands, and the UMCG, Tuberculosis Center Beatrixoord, Haren, The Netherlands. Forty-one adult patients were included after providing written informed consent. The included patients received isoniazid $5 \mathrm{mg} / \mathrm{kg}$, rifampicin $10 \mathrm{mg} / \mathrm{kg}$, ethambutol $15 \mathrm{mg} / \mathrm{kg}$ and pyrazinamide $25 \mathrm{mg} /$ $\mathrm{kg}$. A full PK curve was recorded during the intensive phase of TB treatment after steady state had been reached ( $\geq 2$ weeks). Patients refrained from food intake from $23 \mathrm{~h}$ on the day preceding the PK assessment until $4 \mathrm{~h}$ after intake of the study medication. Serial venous blood samples were collected just prior to and at 1, 1.5, 2, 2.5, 3, 4, 6, 8, 12 and $24 \mathrm{~h}$ after witnessed intake of the study medication. Plasma was separated immediately, frozen at $-80^{\circ} \mathrm{C}$ and transported on dry ice for bioanalysis. Plasma concentrations of rifampicin, ethambutol and pyrazinamide were analysed at the University Medical Centre Nijmegen using validated high-performance liquid chromatography (HPLC) methods as described previously [27]. Plasma concentrations of isoniazid were determined at the UMCG using the validated LC-MS/MS method mentioned earlier [20]. The AUC 
24 ,calculated was calculated using WinNonlin (further information can be found in the original study [16]), while the $\mathrm{AUC}_{24, \text { predicted }}$ was calculated using the best-performing OSS formula in the fasting condition. For the external group, the same PPE $<15 \%$ was considered acceptable, and the number of patients with a PPE $<15 \%$ was calculated.

\section{Results}

Patient characteristics for both groups are presented in Table 1. The assumptions of linear regression for OSS analysis were fulfilled. Table 2 shows the best-performing one, two, and three time-point equations of the four first-line TB drugs individually when administered intravenously or in fasted or fed conditions. Only imprecision of the AUC prediction of ethambutol in the fed condition was higher than the preset limit of $15 \%$. Table 3 presents the best-performing equations for the simultaneous prediction of exposure of all drugs when administered in different conditions, i.e. either intravenously or fasting or fed conditions. Again, only imprecision of ethambutol in the fed condition did not meet the requirements. The time points 0,5 , and $8 \mathrm{~h}$ postdose showed the best prediction for intravenous administration of isoniazid, rifampicin and ethambutol. In the fed condition, sampling times at 1, 2 and $8 \mathrm{~h}$ post-dose were the best predictive factors, while in the fasting condition, sampling time points at 2,4 , and $8 \mathrm{~h}$ post-dose provided the best prediction.

Table 4 shows the best-performing equations for simultaneous predictions of all first-line drugs administered orally, either in the fasting or fed condition. It can be observed that

Table 1 Patient characteristics

\begin{tabular}{|c|c|c|c|}
\hline Characteristic & OSS group $(n=20)$ & $\begin{array}{l}\text { External validation group } \\
(n=37)\end{array}$ & $P$ value \\
\hline Male/female $[n / n(\% / \%)]$ & $12 / 8(60 / 40)$ & $31 / 6(84 / 16)$ & $<0.001^{\mathrm{a}}$ \\
\hline Age, years & $37.0(24.0-51.8)$ & $42.0(30.0-56.5)$ & 0.219 \\
\hline Bodyweight (kg) & $41.5(38.3-47.8)$ & $64(53.2-69.4)$ & $<0.001$ \\
\hline BMI $\left(\mathrm{kg} / \mathrm{m}^{2}\right)$ & $17.0(15.9-19.1)$ & $20.9(18.1-23.6)^{\mathrm{c}}$ & $<0.001$ \\
\hline Underweight $[n(\%)]^{\mathrm{b}}$ & $14(70)$ & $11(30)$ & $0.500^{\mathrm{a}}$ \\
\hline Ethnicity $[n(\%)]$ & & & $<0.001^{\mathrm{a}}$ \\
\hline Asian & $20(100)$ & $5(14)$ & \\
\hline Black & $0(0)$ & $12(32)$ & \\
\hline Caucasian & $0(0)$ & $11(30)$ & \\
\hline Middle Eastern & $0(0)$ & $5(14)$ & \\
\hline Other/mixed origin & $0(0)$ & $4(11)$ & \\
\hline Comorbidity $[n(\%)]$ & $4(20)$ & $14(38)$ & $0.005^{\mathrm{a}}$ \\
\hline Comedication $[n(\%)]$ & $8(40)$ & $29(78)$ & $0.024^{\mathrm{a}}$ \\
\hline \multicolumn{4}{|l|}{ Drug } \\
\hline Isoniazid $(n)$ & 20 & 8 & 0.263 \\
\hline Dose (mg/kg) & $5.4(4.7-5.7)$ & $4.7(4.2-5.8)$ & \\
\hline Dose (mg) & $225(225-225)$ & $300(300-300)$ & \\
\hline Rifampicin $(n)$ & 20 & 31 & 0.070 \\
\hline Dose (mg/kg) & $10.7(9.5-11.4)$ & $9.3(8.6-11.0)$ & \\
\hline Dose (mg) & $450(450-450)$ & $600(600-600)$ & \\
\hline Ethambutol $(n)$ & 20 & 14 & 0.119 \\
\hline Dose $(\mathrm{mg} / \mathrm{kg})$ & $19.6(17.3-20.9)$ & $20.3(18.7-23.6)$ & \\
\hline Dose (mg) & $825(825-825)$ & $1200(1000-1600)$ & \\
\hline Pyrazinamide $(n)$ & 20 & 17 & 0.647 \\
\hline Dose (mg/kg) & $28.6(25.1-30.4)$ & $28.8(27.9-29.6)$ & \\
\hline Dose (mg) & $1200(1200-1200)$ & $2000(1500-2000)$ & \\
\hline
\end{tabular}

Data are expressed as median (IQR) unless otherwise specified

Tested using the Mann-Whitney $U$ test

OSS optimal sampling strategy, IQR interquartile range, $B M I$ body mass index

${ }^{a}$ Tested using the Chi-square test

${ }^{\mathrm{b}}$ Defined as BMI $<18.5 \mathrm{~kg} / \mathrm{m}^{2}$

${ }^{\mathrm{c}} n=35$ 
Table 2 Prediction of individual first-line tuberculosis drugs in different conditions

\begin{tabular}{|c|c|c|c|c|c|c|c|c|}
\hline \multicolumn{3}{|c|}{ Sampling time } & \multirow{2}{*}{$\begin{array}{l}\text { Equation } \\
\mathrm{AUC}_{24}=5.08+12.27 \times \mathrm{C}_{5}\end{array}$} & \multirow{2}{*}{$\begin{array}{l}\text { Adj. } R^{2} \\
0.8929\end{array}$} & \multirow{2}{*}{$\begin{array}{l}\text { MPE } \\
-0.58\end{array}$} & \multirow{2}{*}{$\begin{array}{l}\text { MPPE }(\%) \\
-4.53\end{array}$} & \multirow{2}{*}{$\begin{array}{c}\text { RMSE } \\
1.69\end{array}$} & \multirow{2}{*}{$\frac{\text { MAPE (\%) }}{8.95}$} \\
\hline INH & IV & 5 & & & & & & \\
\hline & & 2,8 & $\mathrm{AUC}_{24}=-0.12+4.32 \times \mathrm{C}_{2}+10.30 \times \mathrm{C}_{8}$ & 0.9313 & 0.04 & 0.34 & 1.92 & 11.46 \\
\hline & & $2,3,8$ & $\mathrm{AUC}_{24}=-0.59+7.34 \times \mathrm{C}_{2}-5.39 \times \mathrm{C}_{3}+14.90 \times \mathrm{C}_{8}$ & 0.9358 & 0.14 & 1.70 & 2.43 & 13.44 \\
\hline & Fasted & 8 & $\mathrm{AUC}_{24}=8.19+13.81 \times \mathrm{C}_{8}$ & 0.9148 & -0.61 & -4.65 & 1.86 & 11.52 \\
\hline & & 1,8 & $\mathrm{AUC}_{24}=5.39+0.93 \times \mathrm{C}_{1}+12.75 \times \mathrm{C}_{8}$ & 0.9378 & 0.31 & 1.88 & 1.46 & 9.53 \\
\hline & & $1,6,8$ & $\mathrm{AUC}_{24}=4.87+1.59 \times \mathrm{C}_{1}-6.82 \times \mathrm{C}_{6}+19.47 \times \mathrm{C}_{8}$ & 0.9625 & 0.49 & 1.71 & 1.41 & 11.46 \\
\hline & Fed & 8 & $\mathrm{AUC}_{24}=3.25+16.13 \times \mathrm{C}_{8}$ & 0.8330 & 0.65 & 6.42 & 3.52 & 23.60 \\
\hline & & 2,8 & $\mathrm{AUC}_{24}=-1.45+2.61 \times \mathrm{C}_{2}+14.99 \times \mathrm{C}_{8}$ & 0.9528 & -0.09 & -2.25 & 1.72 & 14.09 \\
\hline & & $1,2,8$ & $\mathrm{AUC}_{24}=-1.28+1.00 \times \mathrm{C}_{1}+1.93 \times \mathrm{C}_{2}+15.05 \times \mathrm{C}_{8}$ & 0.9638 & -0.44 & -2.53 & 1.19 & 7.90 \\
\hline \multirow[t]{9}{*}{ RIF } & IV & 8 & $\mathrm{AUC}_{24}=-1.02+18.94 \times \mathrm{C}_{8}$ & 0.9402 & -1.60 & -1.77 & 4.60 & 6.71 \\
\hline & & 1,8 & $\mathrm{AUC}_{24}=-8.37+1.15 \times \mathrm{C}_{1}+18.23 \times \mathrm{C}_{8}$ & 0.9541 & -1.14 & -1.31 & 5.96 & 6.37 \\
\hline & & $1,5,8$ & $\mathrm{AUC}_{24}=-2.25+1.24 \times \mathrm{C}_{1}-5.61 \times \mathrm{C}_{5}+25.01 \times \mathrm{C}_{8}$ & 0.9622 & -1.38 & -1.52 & 5.50 & 5.91 \\
\hline & Fasted & 8 & $\mathrm{AUC}_{24}=-5.38+18.91 \times \mathrm{C}_{8}$ & 0.8501 & -2.24 & -5.15 & 7.87 & 9.46 \\
\hline & & 2,8 & $\mathrm{AUC}_{24}=11.78+1.76 \times \mathrm{C}_{2}+16.73 \times \mathrm{C}_{8}$ & 0.8823 & -1.44 & -1.48 & 3.46 & 6.23 \\
\hline & & $2,5,8$ & $\mathrm{AUC}_{24}=-2.95+2.29 \times \mathrm{C}_{2}-4.88 \times \mathrm{C}_{5}+20.97 \times \mathrm{C}_{8}$ & 0.9026 & -0.19 & -0.39 & 4.04 & 6.61 \\
\hline & Fed & 8 & $\mathrm{AUC}_{24}=2.02+14.30 \times \mathrm{C}_{8}$ & 0.8373 & 1.14 & 3.04 & 8.11 & 11.20 \\
\hline & & 2,8 & $\mathrm{AUC}_{24}=-9.67+2.10 \times \mathrm{C}_{2}+14.76 \times \mathrm{C}_{8}$ & 0.9605 & -1.18 & -1.31 & 2.56 & 4.59 \\
\hline & & $2,3,8$ & $\mathrm{AUC}_{24}=-15.18+1.58 \times \mathrm{C}_{2}+0.99 \times \mathrm{C}_{3}+15.05 \times \mathrm{C}_{8}$ & 0.9636 & -0.89 & -1.14 & 5.10 & 7.90 \\
\hline \multirow[t]{9}{*}{ EMB } & IV & 6 & $\mathrm{AUC}_{24}=-1.96+27.75 \times \mathrm{C}_{6}$ & 0.7856 & -0.65 & -3.54 & 1.62 & 13.96 \\
\hline & & 0,8 & $\mathrm{AUC}_{24}=-2.96-19.86 \times \mathrm{C}_{0}+41.52 \times \mathrm{C}_{8}$ & 0.8364 & -0.32 & -3.13 & 2.30 & 14.77 \\
\hline & & $0,5,8$ & $\mathrm{AUC}_{24}=-4.67-21.39 \times \mathrm{C}_{0}+13.65 \times \mathrm{C}_{5}+22.41 \times \mathrm{C}_{8}$ & 0.9105 & 0.11 & 0.73 & 1.50 & 10.80 \\
\hline & Fasted & 8 & $\mathrm{AUC}_{24}=2.75+17.67 \times \mathrm{C}_{8}$ & 0.7332 & -0.19 & -1.37 & 2.24 & 14.19 \\
\hline & & 2,8 & $\mathrm{AUC}_{24}=0.19+1.30 \times \mathrm{C}_{2}+17.61 \times \mathrm{C}_{8}$ & 0.8019 & 0.51 & 3.77 & 2.25 & 14.46 \\
\hline & & $2,4,8$ & $\begin{array}{l}\mathrm{AUC}_{24}=-1.74+1.09 \times \mathrm{C}_{2} \\
+2.29 \times \mathrm{C}_{4}+15.05 \times \mathrm{C}_{8}\end{array}$ & 0.8445 & 0.43 & 3.30 & 2.18 & 12.91 \\
\hline & Fed & 8 & $\mathrm{AUC}_{24}=6.57+10.74 \times \mathrm{C}_{8}$ & 0.3755 & 0.13 & 1.02 & 3.90 & 28.14 \\
\hline & & 2,8 & $\mathrm{AUC}_{24}=-2.01+3.54 \times \mathrm{C}_{2}+13.98 \times \mathrm{C}_{8}$ & 0.5698 & -0.23 & -1.96 & 2.34 & 17.51 \\
\hline & & $1,2,8$ & $\mathrm{AUC}_{24}=-2.14-3.02 \times \mathrm{C}_{1}+4.86 \times \mathrm{C}_{2}+13.77 \times \mathrm{C}_{8}$ & 0.5814 & -0.38 & -2.44 & 2.82 & 18.13 \\
\hline \multirow[t]{6}{*}{ PZA } & Fasted & 8 & $\mathrm{AUC}_{24}=-24.64+20.33 \times \mathrm{C}_{8}$ & 0.9689 & -0.66 & -0.16 & 12.35 & 3.27 \\
\hline & & 5,8 & $\mathrm{AUC}_{24}=-42.50+3.83 \times \mathrm{C}_{5}+16.38 \times \mathrm{C}_{8}$ & 0.9731 & -2.39 & -0.36 & 12.02 & 2.29 \\
\hline & & $0,5,8$ & $\mathrm{AUC}_{24}=4.78 \times \mathrm{C}_{0}+4.47 \times \mathrm{C}_{5}+13.96 \times \mathrm{C}_{8}$ & 0.9752 & 0.02 & 0.02 & 11.75 & 3.02 \\
\hline & Fed & 8 & $\mathrm{AUC}_{24}=4.98+17.79 \times \mathrm{C}_{8}$ & 0.8868 & 0.95 & 0.19 & 27.39 & 6.73 \\
\hline & & 2,8 & $\mathrm{AUC}_{24}=-28.69+2.60 \times \mathrm{C}_{2}+15.83 \times \mathrm{C}_{8}$ & 0.9454 & 1.89 & 0.58 & 19.34 & 3.68 \\
\hline & & $0,2,8$ & $\mathrm{AUC}_{24}=-17.07+8.34 \times \mathrm{C}_{0}+2.81 \times \mathrm{C}_{2}+13.20 \times \mathrm{C}_{8}$ & 0.9710 & 0.73 & 0.16 & 15.28 & 2.81 \\
\hline
\end{tabular}

$M P E$ median prediction error, MPPE median percentage prediction error, $R M S E$ root median squared prediction error, MAPE median absolute percentage prediction error, $A U C 24$ area under the concentration-time curve from time zero to $24 \mathrm{~h}$, Adj. adjusted, $I N H$ isoniazid, $R I F$ rifampicin, $E M B$ ethambutol, $P Z A$ pyrazinamide, $I V$ intravenously

after oral administration, $\mathrm{C}_{2}, \mathrm{C}_{4}$ and $\mathrm{C}_{8}$ are nicely distributed over the absorption, distribution and elimination phase, leading to an adequate $\mathrm{AUC}_{24}$ estimation. The bias and imprecision were acceptable for almost all drugs. We did not calculate the best OSS combined for all three conditions because clinicians will only occasionally combine oral and intravenous administrations. However, when we tested this best-performing OSS $\left(\mathrm{OSS}_{2-4-8}\right)$ following intravenous administration, we found that this OSS showed bias and imprecision that was comparable with $\mathrm{OSS}_{0-5-8}$, indicating that it was also suitable for accurately estimating the $\mathrm{AUC}_{24}$ for intravenous isoniazid, rifampicin and ethambutol (Table 3). Thus, $\mathrm{OSS}_{2-4-8}$ can be used for either oral or intravenous administration.

Table 5 shows the median PPE (range) and number of patients with a PPE $<15 \%$. We showed that $\geq 70 \%$ of patients in the OSS group (for all drugs and oral conditions) had an acceptable PPE value, except for ethambutol in the fed condition. In the external validation group, $\mathrm{OSS}_{2-4-8}$ could correctly predict $79 \%$ and $88 \%$ of the individual $\mathrm{AUC}_{24}$ of ethambutol and pyrazinamide, respectively, but in only $50 \%$ and $23 \%$ of the individual $\mathrm{AUC}_{24}$ of isoniazid and rifampicin, respectively. 
Table 3 Simultaneous prediction of all first-line tuberculosis drugs in different conditions

\begin{tabular}{|c|c|c|c|c|c|c|c|c|}
\hline \multicolumn{3}{|c|}{ Sampling time } & \multirow{2}{*}{$\begin{array}{l}\text { Equation } \\
\mathrm{AUC}_{24}=5.08+12.27 \times \mathrm{C}_{5}\end{array}$} & \multirow{2}{*}{$\begin{array}{c}\text { Adj. } \mathrm{R}^{2} \\
0.8929\end{array}$} & \multirow{2}{*}{$\begin{array}{l}\text { MPE } \\
-0.58\end{array}$} & \multirow{2}{*}{$\begin{array}{l}\text { MPPE (\%) } \\
-4.53\end{array}$} & \multirow{2}{*}{$\begin{array}{c}\text { RMSE } \\
1.69\end{array}$} & \multirow{2}{*}{$\frac{\text { MAPE (\%) }}{8.95}$} \\
\hline IV & 5 & INH & & & & & & \\
\hline & & RIF & $\mathrm{AUC}_{24}=-9.52+14.08 \times \mathrm{C}_{5}$ & 0.8228 & -2.86 & -3.59 & 9.53 & 10.08 \\
\hline & & EMB & $\mathrm{AUC}_{24}=-2.06+22.74 \times \mathrm{C}_{5}$ & 0.7799 & -0.59 & -4.20 & 1.81 & 15.58 \\
\hline & 2,8 & INH & $\mathrm{AUC}_{24}=-0.12+4.32 \times \mathrm{C}_{2}+10.30 \times \mathrm{C}_{8}$ & 0.9313 & 0.04 & 0.34 & 1.92 & 11.46 \\
\hline & & RIF & $\mathrm{AUC}_{24}=-5.34+0.66 \times \mathrm{C}_{2}+18.27 \times \mathrm{C}_{8}$ & 0.9385 & -2.46 & -3.25 & 5.11 & 5.74 \\
\hline & & EMB & $\mathrm{AUC}_{24}=-1.32+1.21 \times \mathrm{C}_{2}+27.59 \times \mathrm{C}_{8}$ & 0.7961 & -0.93 & -8.72 & 2.14 & 15.07 \\
\hline & $0,5,8$ & INH & $\mathrm{AUC}_{24}=4.62+1.21 \times \mathrm{C}_{0}+14.00 \times \mathrm{C}_{5}+2.74 \times \mathrm{C}_{8}$ & 0.8825 & -0.62 & -3.27 & 1.68 & 14.38 \\
\hline & & RIF & $\begin{array}{l}\mathrm{AUC}_{24}=6.80+7.56 \times \mathrm{C}_{0} \\
-5.69 \times \mathrm{C}_{5}+25.58 \times \mathrm{C}_{8}\end{array}$ & 0.9408 & -1.87 & -2.43 & 5.67 & 7.59 \\
\hline & & EMB & $\mathrm{AUC}_{24}=-4.67-21.39 \times \mathrm{C}_{0}+13.65 \times \mathrm{C}_{5}+22.41 \times \mathrm{C}_{8}$ & 0.9105 & 0.11 & 0.73 & 1.50 & 10.80 \\
\hline & $2,4,8$ & INH & $\mathrm{AUC}_{24}=-1.10+5.59 \times \mathrm{C}_{2}-3.00 \times \mathrm{C}_{4}+13.07 \times \mathrm{C}_{8}$ & 0.9308 & -0.10 & -0.71 & 1.97 & 9.44 \\
\hline & & RIF & $\mathrm{AUC}_{24}=-1.43+0.96 \times \mathrm{C}_{2}-2.75 \times \mathrm{C}_{4}+21.22 \times \mathrm{C}_{8}$ & 0.9395 & -2.34 & -2.59 & 4.34 & 4.79 \\
\hline & & EMB & $\mathrm{AUC}_{24}=-3.10+1.22 \times \mathrm{C}_{2}+7.80 \times \mathrm{C}_{4}+14.24 \times \mathrm{C}_{8}$ & 0.8128 & -0.97 & -7.26 & 1.68 & 12.03 \\
\hline \multirow[t]{12}{*}{ Fasted } & 8 & INH & $\mathrm{AUC}_{24}=8.19+13.81 \times \mathrm{C}_{8}$ & 0.9148 & -0.61 & -4.65 & 1.86 & 11.52 \\
\hline & & RIF & $\mathrm{AUC}_{24}=-5.38+18.91 \times \mathrm{C}_{8}$ & 0.8501 & -2.24 & -5.15 & 7.87 & 9.46 \\
\hline & & EMB & $\mathrm{AUC}_{24}=2.75+17.67 \times \mathrm{C}_{8}$ & 0.7332 & -0.19 & -1.37 & 14.12 & 14.19 \\
\hline & & PZA & $\mathrm{AUC}_{24}=-24.64+20.33 \times \mathrm{C}_{8}$ & 0.9689 & -0.66 & -0.16 & 2.48 & 3.27 \\
\hline & 2,8 & INH & $\mathrm{AUC}_{24}=4.83+1.41 \times \mathrm{C}_{2}+11.71 \times \mathrm{C}_{8}$ & 0.9217 & -0.69 & -3.17 & 1.72 & 14.70 \\
\hline & & RIF & $\mathrm{AUC}_{24}=-11.78+1.76 \times \mathrm{C}_{2}+16.73 \times \mathrm{C}_{8}$ & 0.8823 & -1.44 & -1.48 & 3.46 & 6.23 \\
\hline & & EMB & $\mathrm{AUC}_{24}=0.19+1.30 \times \mathrm{C}_{2}+17.61 \times \mathrm{C}_{8}$ & 0.8019 & 0.51 & 3.77 & 14.19 & 14.46 \\
\hline & & PZA & $\mathrm{AUC}_{24}=-36.04+0.89 \times \mathrm{C}_{2}+19.35 \times \mathrm{C}_{8}$ & 0.9684 & -1.73 & -0.40 & 2.05 & 2.63 \\
\hline & $2,4,8$ & INH & $\mathrm{AUC}_{24}=3.81+2.99 \times \mathrm{C}_{2}-4.74 \times \mathrm{C}_{4}+17.08 \times \mathrm{C}_{8}$ & 0.9401 & -0.67 & -4.75 & 1.75 & 11.59 \\
\hline & & RIF & $\mathrm{AUC}_{24}=-4.00+2.16 \times \mathrm{C}_{2}-2.21 \times \mathrm{C}_{4}+17.90 \times \mathrm{C}_{8}$ & 0.8861 & -0.66 & -0.86 & 4.64 & 8.88 \\
\hline & & EMB & $\mathrm{AUC}_{24}=-1.74+1.09 \times \mathrm{C}_{2}+2.29 \times \mathrm{C}_{4}+15.05 \times \mathrm{C}_{8}$ & 0.8445 & 0.43 & 3.30 & 13.75 & 12.91 \\
\hline & & PZA & $\mathrm{AUC}_{24}=-34.77+1.08 \times \mathrm{C}_{2}-0.58 \times \mathrm{C}_{4}+19.76 \times \mathrm{C}_{8}$ & 0.9666 & -1.21 & -0.18 & 2.76 & 3.19 \\
\hline \multirow[t]{12}{*}{ Fed } & 8 & INH & $\mathrm{AUC}_{24}=3.25+16.13 \times \mathrm{C}_{8}$ & 0.8330 & 0.65 & 6.42 & 3.52 & 23.60 \\
\hline & & RIF & $\mathrm{AUC}_{24}=2.02+14.30 \times \mathrm{C}_{8}$ & 0.8373 & 1.14 & 3.04 & 8.11 & 11.20 \\
\hline & & EMB & $\mathrm{AUC}_{24}=6.57+10.74 \times \mathrm{C}_{8}$ & 0.3755 & 0.13 & 1.02 & 3.90 & 28.14 \\
\hline & & PZA & $\mathrm{AUC}_{24}=4.98+17.79 \times \mathrm{C}_{8}$ & 0.8868 & 0.95 & 0.19 & 5.83 & 6.73 \\
\hline & 2,8 & INH & $\mathrm{AUC}_{24}=-1.45+2.61 \times \mathrm{C}_{2}+14.99 \times \mathrm{C}_{8}$ & 0.9528 & -0.09 & -2.25 & 1.72 & 14.09 \\
\hline & & RIF & $\mathrm{AUC}_{24}=-9.67+2.10 \times \mathrm{C}_{2}+14.76 \times \mathrm{C}_{8}$ & 0.9605 & -1.18 & -1.31 & 2.56 & 4.59 \\
\hline & & EMB & $\mathrm{AUC}_{24}=-2.01+3.54 \times \mathrm{C}_{2}+13.98 \times \mathrm{C}_{8}$ & 0.5698 & -0.23 & -1.96 & 2.34 & 17.51 \\
\hline & & PZA & $\mathrm{AUC}_{24}=-28.69+2.60 \times \mathrm{C}_{2}+15.83 \times \mathrm{C}_{8}$ & 0.9454 & 1.89 & 0.58 & 4.12 & 3.68 \\
\hline & $1,2,8$ & INH & $\mathrm{AUC}_{24}=-1.28+1.00 \times \mathrm{C}_{1}+1.93 \times \mathrm{C}_{2}+15.05 \times \mathrm{C}_{8}$ & 0.9638 & -0.44 & -2.53 & 1.19 & 7.90 \\
\hline & & RIF & $\mathrm{AUC}_{24}=-9.77-0.08 \times \mathrm{C}_{1}+2.13 \times \mathrm{C}_{2}+14.78 \times \mathrm{C}_{8}$ & 0.9581 & -1.18 & -1.31 & 2.81 & 4.53 \\
\hline & & EMB & $\mathrm{AUC}_{24}=-2.14-3.02 \times \mathrm{C}_{1}+4.86 \times \mathrm{C}_{2}+13.77 \times \mathrm{C}_{8}$ & 0.5814 & -0.38 & -2.44 & 2.82 & 18.13 \\
\hline & & PZA & $\mathrm{AUC}_{24}=-24.71+1.06 \times \mathrm{C}_{1}+1.75 \times \mathrm{C}_{2}+15.94 \times \mathrm{C}_{8}$ & 0.9521 & -2.47 & -0.50 & 16.31 & 4.17 \\
\hline
\end{tabular}

$M P E$ median prediction error, MPPE median percentage prediction error, $R M S E$ root median squared prediction error, MAPE median absolute percentage prediction error, $A U C 24$ area under the concentration-time curve from time zero to $24 \mathrm{~h}, A d j$. adjusted, $I N H$ isoniazid, $R I F$ rifampicin, $E M B$ ethambutol, $P Z A$ pyrazinamide, $I V$ intravenously

\section{Discussion}

To our knowledge, this is the first study to develop an OSS for the prediction of $\mathrm{AUC}_{24}$ of first-line anti-TB drugs in early treatment and in different conditions. $\mathrm{OSS}_{2-4-8}$ accurately estimated $\mathrm{AUC}_{24}$ for first-line anti-TB drugs after oral administration in early treatment.

If we compare our data with the only study that described an OSS for all first-line anti-TB drugs, several differences can be observed (Table 6) [16]. Apart from the differences already mentioned in the Introduction on feeding conditions and time restrictions, the earlier study included a more variable population with regard to ethnicity and patients from referral TB centres. Meanwhile, in this study, we included only regular outpatients of Asian ethnicity (Table 1).

With regard to isoniazid, Cojutti et al. determined and validated an OSS at 1,2 and $9 \mathrm{~h}$ after dosing in a large population, consisting of 100 patients for the modelling group 
Table 4 Simultaneous prediction of all first-line tuberculosis drugs in fasting and fed conditions

\begin{tabular}{|c|c|c|c|c|c|c|c|c|}
\hline \multicolumn{3}{|c|}{ Sampling time } & \multirow{2}{*}{$\begin{array}{l}\text { Equation } \\
\mathrm{AUC}_{24}=8.19+13.81 \times \mathrm{C}_{8}\end{array}$} & \multirow{2}{*}{$\begin{array}{c}\text { Adj. } R^{2} \\
0.9149\end{array}$} & \multirow{2}{*}{$\begin{array}{l}\text { MPE } \\
-0.61\end{array}$} & \multirow{2}{*}{$\frac{\operatorname{MPPE}(\%)}{-4.65}$} & \multirow{2}{*}{$\begin{array}{c}\text { RMSE } \\
1.86\end{array}$} & \multirow{2}{*}{$\begin{array}{l}\text { MAPE (\%) } \\
11.52\end{array}$} \\
\hline INH & 8 & Fasted & & & & & & \\
\hline & & Fed & $\mathrm{AUC}_{24}=3.25+16.13 \times \mathrm{C}_{8}$ & 0.8330 & 0.65 & 6.42 & 3.52 & 23.60 \\
\hline & 2,8 & Fasted & $\mathrm{AUC}_{24}=4.83+1.41 \times \mathrm{C}_{2}+11.71 \times \mathrm{C}_{8}$ & 0.9218 & -0.69 & -3.17 & 1.72 & 14.70 \\
\hline & & Fed & $\mathrm{AUC}_{24}=-1.45+2.61 \times \mathrm{C}_{2}+14.99 \times \mathrm{C}_{8}$ & 0.9528 & -0.09 & -2.25 & 1.72 & 14.09 \\
\hline & $2,4,8$ & Fasted & $\mathrm{AUC}_{24}=3.81+2.99 \times \mathrm{C}_{2}-4.74 \times \mathrm{C}_{4}+17.08 \times \mathrm{C}_{8}$ & 0.9401 & -0.67 & -4.75 & 1.75 & 11.59 \\
\hline & & Fed & $\mathrm{AUC}_{24}=-2.71+2.36 \times \mathrm{C}_{2}+2.28 \times \mathrm{C}_{4}+12.21 \times \mathrm{C}_{8}$ & 0.9581 & -0.16 & 1.49 & 1.53 & 11.37 \\
\hline \multirow[t]{6}{*}{ RIF } & 8 & Fasted & $\mathrm{AUC}_{24}=-5.38+18.91 \times \mathrm{C}_{8}$ & 0.8501 & -2.24 & -5.15 & 7.87 & 9.46 \\
\hline & & Fed & $\mathrm{AUC}_{24}=2.02+14.30 \times \mathrm{C}_{8}$ & 0.8373 & 1.14 & 3.04 & 8.11 & 11.20 \\
\hline & 2,8 & Fasted & $\mathrm{AUC}_{24}=-11.78+1.76 \times \mathrm{C}_{2}+16.73 \times \mathrm{C}_{8}$ & 0.8823 & -1.44 & -1.48 & 3.46 & 6.23 \\
\hline & & Fed & $\mathrm{AUC}_{24}=-9.67+2.10 \times \mathrm{C}_{2}+14.76 \times \mathrm{C}_{8}$ & 0.9605 & -1.18 & -1.31 & 2.56 & 4.59 \\
\hline & $2,4,8$ & Fasted & $\mathrm{AUC}_{24}=-4.00+2.16 \times \mathrm{C}_{2}-2.21 \times \mathrm{C}_{4}+17.90 \times \mathrm{C}_{8}$ & 0.8861 & -0.66 & -0.86 & 4.64 & 8.88 \\
\hline & & Fed & $\mathrm{AUC}_{24}=-14.57+1.80 \times \mathrm{C}_{2}+1.03 \times \mathrm{C}_{4}+14.55 \times \mathrm{C}_{8}$ & 0.9625 & -0.42 & -0.52 & 2.78 & 6.60 \\
\hline \multirow[t]{6}{*}{ EMB } & 8 & Fasted & $\mathrm{AUC}_{24}=2.75+17.66 \times \mathrm{C}_{8}$ & 0.7333 & -0.19 & -1.37 & 2.24 & 14.19 \\
\hline & & Fed & $\mathrm{AUC}_{24}=6.57+10.74 \times \mathrm{C}_{8}$ & 0.3755 & 0.13 & 1.02 & 3.90 & 28.14 \\
\hline & 2,8 & Fasted & $\mathrm{AUC}_{24}=0.19+1.30 \times \mathrm{C}_{2}+17.61 \times \mathrm{C}_{8}$ & 0.8019 & 0.51 & 3.77 & 2.25 & 14.46 \\
\hline & & Fed & $\mathrm{AUC}_{24}=-2.01+3.54 \times \mathrm{C}_{2}+13.98 \times \mathrm{C}_{8}$ & 0.5698 & -0.23 & -1.96 & 2.34 & 17.51 \\
\hline & $2,4,8$ & Fasted & $\mathrm{AUC}_{24}=-1.74+1.08 \times \mathrm{C}_{2}+2.29 \times \mathrm{C}_{4}+15.05 \times \mathrm{C}_{8}$ & 0.8445 & 0.43 & 3.30 & 2.18 & 12.91 \\
\hline & & Fed & $\mathrm{AUC}_{24}=-2.76+3.33 \times \mathrm{C}_{2}+1.04 \times \mathrm{C}_{4}+12.79 \times \mathrm{C}_{8}$ & 0.5512 & 0.20 & 0.60 & 2.63 & 20.67 \\
\hline \multirow[t]{6}{*}{ PZA } & 8 & Fasted & $\mathrm{AUC}_{24}=-24.64+20.33 \times \mathrm{C}_{8}$ & 0.9689 & -0.66 & -0.16 & 12.35 & 3.27 \\
\hline & & Fed & $\mathrm{AUC}_{24}=4.98+17.79 \times \mathrm{C}_{8}$ & 0.8868 & 0.95 & 0.19 & 27.39 & 6.73 \\
\hline & 2,8 & Fasted & $\mathrm{AUC}_{24}=-36.04+0.89 \times \mathrm{C}_{2}+19.35 \times \mathrm{C}_{8}$ & 0.9684 & -1.73 & -0.40 & 10.20 & 2.63 \\
\hline & & Fed & $\mathrm{AUC}_{24}=-28.69+2.60 \times \mathrm{C}_{2}+15.83 \times \mathrm{C}_{8}$ & 0.9454 & 1.89 & 0.58 & 19.34 & 3.68 \\
\hline & $2,4,8$ & Fasted & $\mathrm{AUC}_{24}=-34.77+1.08 \times \mathrm{C}_{2}-0.58 \times \mathrm{C}_{4}+19.76 \times \mathrm{C}_{8}$ & 0.9666 & -1.21 & -0.18 & 13.74 & 3.19 \\
\hline & & Fed & $\mathrm{AUC}_{24}=-2.34+3.13 \times \mathrm{C}_{2}-2.91 \times \mathrm{C}_{4}+18.07 \times \mathrm{C}_{8}$ & 0.9485 & -0.27 & -0.11 & 21.32 & 4.28 \\
\hline
\end{tabular}

$M P E$ median prediction error, MPPE median percentage prediction error, $R M S E$ root median squared prediction error, $M A P E$ median absolute percentage prediction error, $A U C 24$ area under the concentration-time curve from time zero to $24 \mathrm{~h}, A d j$. adjusted, $I N H$ isoniazid, $R I F$ rifampicin, $E M B$ ethambutol, $P Z A$ pyrazinamide

and 85 for the validation group [17] (Table 6). Our proposed time point of $8 \mathrm{~h}$ post-dose is comparable with that of Cojutti et al., indicating that a rather late time point is capable of better predicting $\mathrm{AUC}_{24}$ [17]. In another publication by the same group, a threshold of $55 \mathrm{mg} \times \mathrm{h} / \mathrm{L}$ for toxicity of isoniazid has been proposed [28]. In none of our patients, in either the OSS or external validation groups, was such a high AUC ${ }_{24}$ observed, possibly due to the fact that the majority of the included patients were fast acetylators.

Rifampicin shows auto-induction that is complete after 20-30 days [29]. Auto-induction might influence the OSS as PK properties may change. This was indeed shown in our OSS group experiencing a higher AUC ${ }_{24}$ (range $36-129 \mathrm{mg} \times \mathrm{h} / \mathrm{L}$ ) than $\mathrm{AUC}_{24}$ in steady-state patients [16]. However, the $\mathrm{AUC}_{24}$ was not in the range (166-321 $\mathrm{mg} \times \mathrm{h} / \mathrm{L})$ of the high dose rifampicin, i.e. up to $35 \mathrm{mg} / \mathrm{kg}$ [30]. The external validation using steady-state patients did not succeed for rifampicin as the majority of patients showed a PPE $>15 \%$ (Table 5). It is apparently difficult to predict the complex PK of rifampicin based on early measurements. For this drug, we suggest using an OSS based on a population PK model [15].

Regarding the internal jackknife validation, the prediction of ethambutol was the most difficult and $\mathrm{OSS}_{2-4-8}$ was unable to accurately predict a high percentage of individual AUCs (Table 5). However, TDM of ethambutol is rarely performed, and if performed, it is usually in cases of decreased renal function, trying to limit toxicity [11]. Following on from this, ethambutol is mainly used to prevent the emergence of resistance developing for isoniazid and rifampicin. We believe the lower precision in the prediction of $\mathrm{AUC}_{24}$ of ethambutol is acceptable in daily practice. It was striking that $\mathrm{OSS}_{2-4-8}$ predicted $\mathrm{AUC}_{24}$ of ethambutol rather well in the external validation group (Table 5).

The $\mathrm{AUC}_{24}$ of pyrazinamide has not been linked to toxicity [31]. Although the relationship between pyrazinamide dose and hepatotoxicity remains debatable, it appears that doses over $50 \mathrm{mg} / \mathrm{kg}$ may compromise patient safety. Current dosing of pyrazinamide also appears to be on the lower side of effective [32]. $\mathrm{OSS}_{2-4-8}$ well-predicted the $\mathrm{AUC}_{24}$ of pyrazinamide in the external validation group, although the 
Table 5 Median percentage prediction error (range) and number of patients (\%) with a PPE $<15 \%$ for OSS and external validation groups

\begin{tabular}{|c|c|c|c|c|c|}
\hline \multirow[t]{2}{*}{ Condition } & \multirow[t]{2}{*}{$\mathrm{OSS}_{2-4-8}$ equation } & \multicolumn{2}{|l|}{ OSS group } & \multicolumn{2}{|l|}{ External validation group } \\
\hline & & $\begin{array}{l}\text { PPE \% [median } \\
\text { (range)] }\end{array}$ & $\begin{array}{l}\text { No. of patients } \\
\text { with PPE }<15 \% \\
(\%)\end{array}$ & PPE \% [median (range)] & $\begin{array}{l}\text { No. of patients } \\
\text { with PPE }<15 \% \\
(\%)\end{array}$ \\
\hline \multicolumn{6}{|l|}{ Isoniazid } \\
\hline Fasted & $3.81+2.99 \times \mathrm{C}_{2}-4.74 \times \mathrm{C}_{4}+17.08 \times \mathrm{C}_{8}$ & $9.8(0.1-40.7)$ & $14 / 20(70)$ & $14.7(4.7-28.9)$ & $4 / 8(50)$ \\
\hline Fed & $-2.71+2.36 \times \mathrm{C}_{2}+2.28 \times \mathrm{C}_{4}+12.21 \times \mathrm{C}_{8}$ & $9.3(0.5-46.6)$ & $16 / 20(80)$ & NA & NA \\
\hline \multicolumn{6}{|l|}{ Rifampicin } \\
\hline Fasted & $-4.00+2.16 \times \mathrm{C}_{2}-2.21 \times \mathrm{C}_{4}+17.90 \times \mathrm{C}_{8}$ & $7.6(0.0-36.3)$ & $18 / 20(90)$ & $38(2.1-108.2)$ & $7 / 31(23)$ \\
\hline Fed & $-14.57+1.80 \times \mathrm{C}_{2}+1.03 \times \mathrm{C}_{4}+14.55 \times \mathrm{C}_{8}$ & $5.8(0.0-26.8)$ & $16 / 20(80)$ & NA & NA \\
\hline \multicolumn{6}{|c|}{ Ethambutol } \\
\hline Fasted & $-1.74+1.09 \times \mathrm{C}_{2}+2.29 \times \mathrm{C}_{4}+15.05 \times \mathrm{C}_{8}$ & $10.7(2.8-21.8)$ & $14 / 20(70)$ & $12(1.4-66.7)$ & $11 / 14(79)$ \\
\hline Fed & $-2.76+3.33 \times \mathrm{C}_{2}+1.04 \times \mathrm{C}_{4}+12.79 \times \mathrm{C}_{8}$ & $13.5(0.7-166.2)$ & $11 / 20(55)$ & NA & NA \\
\hline \multicolumn{6}{|c|}{ Pyrazinamide } \\
\hline Fasted & $-34.77+1.08 \times \mathrm{C}_{2}-0.58 \times \mathrm{C}_{4}+19.76 \times \mathrm{C}_{8}$ & $2.4(0.1-10.6)$ & $20 / 20(100)$ & $4.2(0.1-41.7)$ & $15 / 17(88)$ \\
\hline Fed & $-2.34+3.13 \times \mathrm{C}_{2}-2.91 \times \mathrm{C}_{4}+18.07 \times \mathrm{C}_{8}$ & $3.4(0.1-9.5)$ & $20 / 20(100)$ & NA & NA \\
\hline
\end{tabular}

$P P E$ percentage prediction error, $O S S$ optimal sampling strategy, $N A$ not applicable

Table 6 Other optimal sampling strategy studies

\begin{tabular}{llll}
\hline Drug & $N$ & OSS, AUC 24 equation & Comment \\
\hline Isoniazid & 12 & $2-4-6\left(-1.46+1.18 \times \mathrm{C}_{2}+4.71 \times \mathrm{C}_{4}+5.51 \times \mathrm{C}_{6}\right)$ & Fasting steady state, \\
Rifampicin & 33 & $2-4-6\left(-2.22+2.05 \times \mathrm{C}_{2}+2.25 \times \mathrm{C}_{4}+4.93 \times \mathrm{C}_{6}\right)$ & internally validated \\
Pyrazinamide & 19 & $2-4-6\left(-48.65+1.78 \times \mathrm{C}_{2}+3.58 \times \mathrm{C}_{4}+9.90 \times \mathrm{C}_{6}\right)$ & jackknife analysis \\
Ethambutol [16] & 19 & $2-4-6\left(-6.34+5.22 \times \mathrm{C}_{2}+2.83 \times \mathrm{C}_{4}+7.07 \times \mathrm{C}_{6}\right)$ & \\
Isoniazid [17] & $100 \mathrm{M}$ & $1-2-5\left(-1.88+1.05 \times \mathrm{C}_{1}+0.78 \times \mathrm{C}_{2}+9.44 \times \mathrm{C}_{5}\right)$ & Fasting steady state \\
& $85 \mathrm{~V}$ & $1-2-9\left(-0.65+1.00 \times \mathrm{C}_{1}+1.94 \times \mathrm{C}_{2}+15.45 \times \mathrm{C}_{9}\right)$ & \\
& & $0.5-2-12\left(0.55+0.76 \times \mathrm{C}_{0.5}+3.15 \times \mathrm{C}_{2}+19.79 \times \mathrm{C}_{12}\right)$ & \\
Rifampicin [15] & 55 & $1-3-8$, Bayesian approach using population PK model & Fasting and fed steady \\
& & & state, internally \\
& & & validated jackknife \\
& & & analysis \\
Rifampicin [18] & $58 \mathrm{M}$ & $2-4-12\left(-2.47+1.92 \times \mathrm{C}_{2}+4.38 \times \mathrm{C}_{4}+13.7 \times \mathrm{C}_{12}\right)$ & Fasting steady state \\
& $25 \mathrm{~V}$ & & \\
\hline
\end{tabular}

$M$ modelling, $V$ validation, $O S S$ optimal sampling strategy, AUC24 area under the concentration-time curve from time zero to $24 \mathrm{~h}, P K$ pharmacokinetic drug had not reached steady state in the first 3 days of treatment. Therefore, $\mathrm{OSS}_{2-4-8}$ can be used to estimate the AUC ${ }_{24}$ of pyrazinamide in other patient groups.

We calculated linear regression equations for OSS for all time points included in the study, for one to three time points, leading to 231 equations per drug and condition. Several equations are comparable in terms of bias and precision and can be used with the same extent of confidence. To be able to widely implement TDM, it would be beneficial to make all equations electronically available, e.g. in an application (app). The user would easily calculate the $\mathrm{AUC}_{24}$ by entering data regarding sampling times, concentration, type of drugs, administration route and condition.

There are several limitations to this study. Although the sample size can be considered standard for intensive PK studies, it was relatively small for an OSS study. To calculate

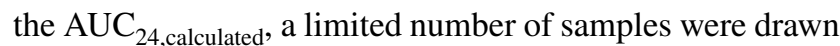
in the elimination phase of the drugs, possibly resulting in an over- or underestimation of the true $\mathrm{AUC}_{24}$. We believe this deviation is acceptable as rich sampling, especially in the elimination phase, is hardly ever performed in daily practice. We internally validated the OSS equations using the jackknife method instead of a prospective validation with new subjects. However, this method is appropriate for small sample sizes and effectively eliminates the major influence of a particular subject [22]. The number of patients $(n=8)$ in the external validation group for isoniazid was too small to draw a conclusion on the external validity of $\mathrm{OSS}_{2-4-8}$. One might argue that OSS should have been developed using validated population PK models. In our opinion, both strategies have 
advantages and disadvantages. A Bayesian approach using a population PK model is more flexible, allowing for deviations from the exact sampling times. A disadvantage is the use of software and use of the model, whereas multiple linear regression formulas yield straightforward equations to fill in [15]. Lastly, sampling took place in the first 3 days of treatment, thus none of the drugs reached steady state. However, we believe that performing TDM early on in the treatment phase could be of importance for isoniazid as this drug is most active in the first 3 days of treatment because of its high early bactericidal activity [33]. If one waits until steady state, precious time is missed to optimize isoniazid treatment [17].

\section{Conclusions}

In the early days of TB treatment, an OSS at 2, 4 and $8 \mathrm{~h}$ post-dose adequately predicted the $\mathrm{AUC}_{24}$ of first-line antiTB drugs in fasting or fed conditions. The external validation group for isoniazid was too small to draw a conclusion, and, for rifampicin, showed that $\mathrm{OSS}_{2-4-8}$ was unable to accurately predict $\mathrm{AUC}_{24}$ in patients in the steady state phase of treatment. $\mathrm{OSS}_{2-4-8}$ was able to predict the $\mathrm{AUC}_{24}$ of ethambutol and pyrazinamide with sufficient accuracy and precision. We believe that our OSS strategy may be a useful tool for optimizing exposure of first-line anti-TB drugs, although welldefined PK/PD targets of efficacy or toxicity are still lacking.

Acknowledgements The authors thank all study participants for their participation in this PK study.

Funding The PK study NCT02121314 was funded by the Directorate General of Higher Education of Indonesia.

\section{Compliance with ethical standards}

Conflicts of Interest Antonia Morita I. Saktiawati, Marcel Harkema, Althaf Setyawan, Yanri W. Subronto, Sumardi, Ymkje Stienstra, Rob E. Aarnoutse, Cecile Magis-Escurra, Jos G.W. Kosterink, Tjip S. van der Werf, Jan-Willem C. Alffenaar and Marieke G.G. Sturkenboom declare that they have no conflicts of interest

Ethical Approval All procedures performed in studies involving human participants were in accordance with the ethical standards of the institutional and/or national research committee and with the 1964 Helsinki declaration and its later amendments or comparable ethical standards.

Informed Consent Informed consent was obtained from all individual participants included in the study.

Author Contributions AMS, MGGS, YS, JWCA, TSW, YWS, S, REA, CM-E and JGWK wrote the manuscript; JWCA, MGGS, TSW, JGWK and AMS designed the research; and MGGS, AMS, MH, AS and JWCA analysed and interpreted the data.

Open Access This article is distributed under the terms of the Creative Commons Attribution-NonCommercial 4.0 International License (http://creativecommons.org/licenses/by-nc/4.0/), which permits any noncommercial use, distribution, and reproduction in any medium, provided you give appropriate credit to the original author(s) and the source, provide a link to the Creative Commons license, and indicate if changes were made.

\section{References}

1. World Health Organization. Global tuberculosis report. Geneva: World Health Organization; 2017.

2. World Health Organization. Guidelines for the treatment of drugsusceptible tuberculosis and patient care. Geneva: World Health Organization; 2017.

3. Pasipanodya JG, McIlleron H, Burger A, Wash PA, Smith P, Gumbo T. Serum drug concentrations predictive of pulmonary tuberculosis outcomes. J Infect Dis. 2013;208:1464-73.

4. Srivastava S, Pasipanodya JG, Meek C, Leff R, Gumbo T. Multidrug-resistant tuberculosis not due to noncompliance but to between-patient pharmacokinetic variability. J Infect Dis. 2011;204:1951-9.

5. McIlleron H, Wash P, Burger A, Norman J, Folb PI, Smith P. Determinants of rifampin, isoniazid, pyrazinamide, and ethambutol pharmacokinetics in a cohort of tuberculosis patients. Antimicrob Agents Chemother. 2006;50:1170-7.

6. Yew WW. Clinically significant interactions with drugs used in the treatment of tuberculosis. Drug Saf. 2002;25:111-33.

7. Müller A, Osório C, Silva D, Sbruzzi G, de Tarso P, Dalcin R. Interventions to improve adherence to tuberculosis treatment: systematic review and meta-analysis. Int J Tuberc Lung Dis. 2018;22:731-40.

8. Alsaad N, Wilffert B, van Altena R, de Lange WC, van der Werf TS, Kosterink JG, et al. Potential antimicrobial agents for the treatment of multidrug-resistant tuberculosis. Eur Respir J. 2014;43:884-97.

9. Chang K, Nuermberger E, Sotgiu G, Leung C. New drugs and regimens for tuberculosis. Respirology. 2018;23:978-90.

10. Gumbo T. New susceptibility breakpoints for first-line antituberculosis drugs based on antimicrobial pharmacokinetic/pharmacodynamic science and population pharmacokinetic variability. Antimicrob Agents Chemother. 2010;54:1484-91.

11. Alsultan A, Peloquin CA. Therapeutic drug monitoring in the treatment of tuberculosis: an update. Drugs. 2014;74:839-54.

12. Zuur MA, Bolhuis MS, Anthony R, den Hertog A, van der Laan T, Wilffert B, et al. Current status and opportunities for therapeutic drug monitoring in the treatment of tuberculosis. Expert Opin Drug Metab Toxicol. 2016;12:509-21.

13. Alffenaar J-W, Tiberi S, Verbeeck RK, Heysell SK, Grobusch MP. Therapeutic drug monitoring in tuberculosis: practical application for physicians. Clin Infect Dis. 2017;64:104-5.

14. Motta I, Calcagno A, Bonora S. Pharmacokinetics and pharmacogenetics of anti-tubercular drugs: a tool for treatment optimization? Expert Opin Drug Metab Toxicol. 2018;14:59-82.

15. Sturkenboom MG, Mulder LW, de Jager A, van Altena R, Aarnoutse RE, de Lange WCM, et al. Pharmacokinetic modeling and optimal sampling strategies for therapeutic drug monitoring of rifampin in patients with tuberculosis. Antimicrob Agents Chemother. 2015;59:4907-13

16. Magis-Escurra C, Later-Nijland HMJ, Alffenaar JWC, Broeders J, Burger DM, van Crevel R, et al. Population pharmacokinetics and limited sampling strategy for first-line tuberculosis drugs and moxifloxacin. Int J Antimicrob Agents. 2014;44:229-34.

17. Cojutti P, Giangreco M, Isola M, Pea F. Limited sampling strategies for determining the area under the plasma concentration-time curve for 
isoniazid might be a valuable approach for optimizing treatment in adult patients with tuberculosis. Int J Antimicrob Agents. 2017;50:23-8.

18. Medellin-Garibay SE, Correa-Lopez T, Romero-Mendez C, Milan-Segovia RC, Romano-Moreno S. Limited sampling strategies to predict the area under the concentration-time curve for rifampicin. Ther Drug Monit. 2014;36:746-51.

19. Saktiawati AM, Sturkenboom MG, Stienstra Y, Subronto YW, Sumardi S, Kosterink JG, et al. Impact of food on the pharmacokinetics of first-line anti-TB drugs in treatment-naive TB patients: a randomized cross-over trial. J Antimicrob Chemother. 2016;71:703-10.

20. Sturkenboom MGG, van der Lijke H, Jongedijk EM, Kok WT, Greijdanus B, Uges DRA, et al. Quantification of isoniazid, pyrazinamide and ethambutol in serum using liquid chromatography-tandem mass spectrometry. J Appl Bioanal. 2015;1:89-98.

21. de Velde F, Alffenaar JW, Wessels AM, Greijdanus B, Uges DR. Simultaneous determination of clarithromycin, rifampicin and their main metabolites in human plasma by liquid chromatography-tandem mass spectrometry. J Chromatogr B. 2009;877:1771-7.

22. Ting LSL, Villeneuve E, Ensom MH. Beyond cyclosporine: a systematic review of limited sampling strategies for other immunosuppressants. Ther Drug Monit. 2006;28:419-30.

23. Barraclough KA, Isbel NM, Kirkpatrick CM, Lee KJ, Taylor PJ, Johnson DW, et al. Evaluation of limited sampling methods for estimation of tacrolimus exposure in adult kidney transplant recipients. Br J Clin Pharmacol. 2011;71:207-23.

24. David OJ, Johnston A. Limited sampling strategies for estimating cyclosporin area under the concentration-time curve: review of current algorithms. Ther Drug Monit. 2001;23:100-14.

25. Sheiner LB, Beal SL. Some suggestions for measuring predictive performance. J Pharmacokinet Biopharm. 1981;9:503-12.
26. Meier-Kriesche HU, Kaplan B, Brannan P, Kahan BD, Portman RJ. A limited sampling strategy for the estimation of eight-hour neoral areas under the curve in renal transplantation. Ther Drug Monit. 1998;20:401-7.

27. Ruslami R, Nijland HM, Alisjahbana B, Parwati I, van Crevel R, Aarnoutse RE. Pharmacokinetics and tolerability of a higher rifampin dose versus the standard dose in pulmonary tuberculosis patients. Antimicrob Agents Chemother. 2007;51:2546-51.

28. Cojutti P, Duranti S, Isola M, Baraldo M, Viale P, Bassetti M, et al. Might isoniazid plasma exposure be a valuable predictor of drug-related hepatotoxicity risk among adult patients with TB? J Antimicrob Chemother. 2016;71:1323-9.

29. Chirehwa MT, Rustomjee R, Mthiyane T, Onyebujoh P, Smith $\mathrm{P}, \mathrm{McIlleron} \mathrm{H}$, et al. Model-based evaluation of higher doses of rifampin using a semimechanistic model incorporating autoinduction and saturation of hepatic extraction. Antimicrob Agents Chemother. 2016;60:487-94.

30. Boeree MJ, Diacon AH, Dawson R, Narunsky K, du Bois J, Venter A, et al. A dose ranging trial to optimize the dose of rifampin in the treatment of tuberculosis. Am J Respir Crit Care Med. 2015;191:1058-65.

31. Gumbo T, Angulo-Barturen I, Ferrer-Bazaga S. Pharmacokineticpharmacodynamic and dose-response relationships of antituberculosis drugs: recommendations and standards for industry and academia. J Infect Dis. 2015;211(Suppl):S96-106.

32. Alsultan A, Savic R, Dooley KE, Weiner M, Whitworth W, MacKenzie WR, et al. Population Pharmacokinetics of Pyrazinamide in Patients with Tuberculosis. Antimicrob Agents Chemother. 2017;61:e02625-7.

33. Diacon AH, Donald PR. The early bactericidal activity of antituberculosis drugs. Expert Rev Anti Infect Ther. 2014;12:223-37.

\section{Affiliations}

\section{Antonia Morita I. Saktiawati ${ }^{1,2,3} \cdot$ Marcel Harkema $^{4} \cdot$ Althaf Setyawan $^{5} \cdot$ Yanri W. Subronto $^{1,3} \cdot$ Sumardi $^{1}$. Ymkje Stienstra $^{6} \cdot$ Rob E. Aarnoutse ${ }^{7}$. Cecile Magis-Escurra ${ }^{8} \cdot$ Jos G. W. Kosterink ${ }^{9,10} \cdot$ Tjip S. van der Werf ${ }^{2,6}$. Jan-Willem C. Alffenaar ${ }^{9,11}$. Marieke G. G. Sturkenboom ${ }^{9}$}

1 Department of Internal Medicine, Faculty of Medicine, Public Health and Nursing, Universitas Gadjah Mada, Yogyakarta, Indonesia

2 Department of Pulmonary Diseases and Tuberculosis, University Medical Center Groningen, University of Groningen, Groningen, The Netherlands

3 Center for Tropical Medicine, Faculty of Medicine, Public Health and Nursing, Universitas Gadjah Mada, Yogyakarta, Indonesia

4 Groningen, The Netherlands

5 Department of Biostatistics, Epidemiology, and Population Health, Faculty of Medicine, Public Health and Nursing, Universitas Gadjah Mada, Yogyakarta, Indonesia

6 Department of Internal Medicine/Infectious Diseases, University Medical Center Groningen, University of Groningen, Groningen, The Netherlands
7 Department of Pharmacy, Radboud University Medical Center, Radboud Institute for Health Sciences, Nijmegen, The Netherlands

8 Department of Pulmonary Diseases, Radboud University Medical Center-Dekkerswald, Groesbeek, The Netherlands

9 Department of Clinical Pharmacy and Pharmacology, University Medical Center Groningen, University of Groningen, PO Box 30.001, 9700 RB Groningen, The Netherlands

10 Unit Pharmacotherapy, Epidemiology and Economy, Groningen Research Institute of Pharmacy, University of Groningen, Groningen, The Netherlands

1 Faculty of Medicine and Health, School of Pharmacy, University of Sydney, Sydney, NSW, Australia 